\title{
Qualidade ambiental e promoção de saúde: o que determina a realização de atividades físicas em parques urbanos?
}

Alba Regina Azevedo Arana* Fernanda Berguerand Xavier ${ }^{* *}$

\section{Resumo}

Evidências encontradas em estudos recentes indicam que a oferta de parques urbanos está associada a benefícios na saúde da população local. Um mecanismo proposto para essa questão é que os parques influenciam positivamente a auto percepção de saúde do indivíduo e incentiva a prática de atividades físicas. No entanto, estudos que exploram essa associação têm produzido resultados mistos. O objetivo deste estudo, portanto, foi revisar evidências documentadas no Brasil e no mundo que associam parques urbanos como fator de qualidade ambiental com o nível de atividade física da população, e propor a seguinte reflexão: em que extensão os parques compreendem uma chave para a redução do sedentarismo e quais as características determinantes desses locais que atuam como promotoras de saúde através da prática de atividades físicas? Concluiu-se que os parques urbanos trazem benefícios à saúde, mas não garantem por si sós o aumento do nível de atividade física. Muitos outros fatores facilitam ou impedem a utilização dos parques de maneira ativa. Pesquisas nacionais que exploram o tema são extremamente escassas e precisam ser incentivadas no para que

* Coordenadora do Programa de Meio Ambiente e Desenvolvimento Regional da UNOESTE (MMADRE). (alba@unoeste.br).

Mestre em Meio Ambiente (Universidade do Oeste Paulista UNOESTE - em Presidente Prudente/SP.). (ernandaberguerand@hotmail.com).

Geosul, Florianópolis, v. 32, n. 63, p 201-228, jan./abr. 2017 
ARANA, A.R.A. \& XAVIER, F.B. Qualidade ambiental e promoção de ... haja maior clareza a respeito desse importante papel dos parques urbanos no Brasil.

Palavras chave: Parques urbanos; Atividade física; Qualidade ambiental; Saúde.

Environmental quality and health promotion: what determines performance of physical activity in urban parks?

\begin{abstract}
The evidence from recent studies indicate that the supply of urban parks is associated with the local population's health benefits. A proposed mechanism for this question is that the parks positively influence the self-perception of the individual's health and encourages physical activity. However, studies exploring this association have produced mixed results. The objective of this study was to review evidences documented in Brazil and in the world that linked urban parks and environmental quality factor with the physical activity level of the population, and propose the following reflection: to what extent the parks comprehend a key to reducing sedentary habits and what are the determining characteristics of those places that act as promoters of health through physical activity? We conclude that urban parks provide health benefits, but do not guarantee increased physical activity level by itself. Many other factors facilitate or impede the use of parks actively. National surveys that explore the theme are extremely scarce and need to be encouraged for clarifying this important role of urban parks in Brazil.
\end{abstract}

Key words: urban parks; physical activity; environmental quality; health.

\title{
Introdução
}

A inatividade física possui uma relação causal com quase $30 \%$ de todas as mortes por cardiopatia, câncer de cólon e diabetes 
ARANA, A.R.A. \& XAVIER, F.B. Qualidade ambiental e promoção de ...

(MCARDLE, 2011). Em qualquer idade, tornar-se fisicamente mais ativo retarda a mortalidade relacionada a essas e outras causas. As pessoas com estilo de vida mais saudáveis sobrevivem por mais tempo, e o risco de incapacitação e de procura por assistência médica é protelado (MCARDLE, 2011).

O sedentarismo é apontado como problema de saúde púbica no Brasil. Dados do Instituto Brasileiro de Geografia Estatística (IBGE) em pesquisa divulgada em Dezembro de 2014 apontam que $46 \%$ da população com idade superior a 18 anos é sedentária, não atingindo a recomendação de 150 minutos de atividade física por semana, seja nos momentos de lazer, no trabalho ou nos deslocamentos até o trabalho (PNS, 2014). Ainda segundo a pesquisa, o percentual de pessoas que praticam atividades físicas no lazer diminui de acordo com a faixa etária. De 18 a 24 anos, a proporção é de 35,3\%. Essas taxas vão caindo para $25,5 \%$ (de 25 a 39 anos), $18,3 \%$ (de 40 a 59 anos) e 13,6\% (de 60 anos ou mais).

Intervenções de saúde direcionadas à mudança comportamental do indivíduo para torna-lo fisicamente ativo têm alcançado mudanças positivas, mas os efeitos têm se mostrado pequenos e de curto prazo, não havendo avanço substancial nos níveis de atividade física da população (BEDIMO-RUNG et al., 2005).

Ademais, pesquisadores têm constatado que a vida em ambientes mais naturais influencia positivamente a auto percepção de saúde das pessoas e leva a um menor risco de mortalidade (MAAS et al., 2008). De acordo com Branas et al. (2011), uma estratégia multinível que combine indivíduo e meio ambiente pode atingir metas maiores e mais sustentáveis, pois influenciam maior número de pessoas por um período de tempo significativamente superior aos programas individuais de saúde.

As grandes concentrações urbanas, a redução dos espaços livres, assim como a revolução tecnológica criaram cenários que predispõem ao estilo de vida inativo (COLLET et al., 2008). Ainda que constitua apenas um único fator, parques urbanos são espaços públicos comuns que podem desempenhar um papel significativo no aumento dos níveis de atividade física da população. Eles 
ARANA, A.R.A. \& XAVIER, F.B. Qualidade ambiental e promoção de ...

fornecem locais propícios para que as pessoas caminhem ou corram, muitas vezes possuem instalações específicas para esportes, exercícios e outras atividades vigorosas, oferecendo a oportunidade de incluir atividades físicas nos momentos de lazer de forma gratuita.

Fredric Olmstead (1999, apud COHEN et al., 2007), o "pai dos parques urbanos" observa que esses devem ser construídos como locais onde os moradores da cidade possam experimentar a beleza da natureza, respirar ar fresco e ter um lugar receptivo para ouvir música e apreciar a arte, praticar atividades esportivas, bem como conviver com amigos e vizinhos. Sendo assim, parques urbanos podem desempenhar um papel na facilitação da atividade física, mas não necessariamente fazê-lo, por também proporcionar oportunidades para que as pessoas se envolvam em comportamento sedentário (WALKER, 2004; apud COHEN et al., 2007).

A escassez de informações quanto à forma de utilização e os fatores que podem facilitar ou impedir a utilização dos parques de maneira ativa, traz à tona a necessidade de compreensão dos fatores que possam intervir na ocupação de parques urbanos enquanto espaço de lazer (COLLET et al., 2008).

Diante destes elementos apresentados temos alguns questionamentos para a reflexão: em que extensão os parques compreendem uma chave para a redução do sedentarismo? Quais as características determinantes desses locais que atuam como promotoras de saúde através da prática de atividades físicas? Como os parques urbanos ajudam na promoção da qualidade de vida das pessoas?

Desta forma, este artigo tem como objetivo apresentar a importância dos parques urbanos para a promoção de saúde e práticas de atividades físicas no espaço urbano. Busca ainda revisar evidências documentadas no Brasil e no mundo que associam parques urbanos como fator de qualidade ambiental com o nível de atividade física da população.

A metodologia utilizada para a realização deste trabalho está fundamentada na pesquisa bibliográfica. Diferentes fontes foram pesquisadas (artigos, teses, dissertações e livros), sendo as 
ARANA, A.R.A. \& XAVIER, F.B. Qualidade ambiental e promoção de ...

fontes primárias identificadas com base no seu título e selecionadas todos os resumos considerados relevantes. A partir disso, todos os artigos selecionados foram obtidos em sua versão integral e então examinados. Por último, realizaram-se consultas às referências bibliográficas e também buscas pelo nome do primeiro autor desses estudos selecionados a fim de identificar outras fontes que estivessem dentro dos requeridos critérios.

Informações sobre quem são os potenciais frequentadores dos parques públicos, o que eles buscam e quais as atividades que realizam nesses ambientes podem elucidar a real contribuição desses locais na estratégia de promoção de saúde bem como potencializar seus benefícios. No entanto, a diversidade de resultados e variáveis encontradas nesses estudos têm produzido resultados mistos (ORD et al., 2013), não concebendo padrões consistentes de associação.

$\mathrm{O}$ texto organizado em quatro partes e uma conclusão. $\mathrm{Na}$ primeira parte são apresentados como a atividade física favorece na promoção a saúde enfocando a epidemiologia da atividade física. Na segunda parte são expostos o conceito de qualidade ambiental urbana, qualidade de vida e qualidade ambiental das cidades salientando suas inter-relações e multidimensionalidade dos conceitos. Na terceira parte estudam-se os fatores utilizados para medir o uso dos parques, tais como: as condições dos parques, facilidade ou dificuldade de acesso, estética do local, segurança percebida, vegetação, acessibilidade entre outros fatores. Na quarta parte são discutidos as razões individuais para utilização dos parques urbanos, enfocando que a utilização dos parques não depende apenas de atributos que o local oferece, mas também se relacionam com preferências individuais como idade, hábitos de exercício e raça/etnia dos frequentadores.

\section{Saúde e atividade fisica}

É importante analisarmos, do ponto de vista da saúde, o potencial que a prática regular de atividades físicas oferece na 
ARANA, A.R.A. \& XAVIER, F.B. Qualidade ambiental e promoção de ...

estratégia de promoção de saúde e qualidade de vida. Para isso, faremos a conceituação de alguns termos utilizados durante este estudo para maior esclarecimento do tema, e também apresentaremos os benefícios comprovados pela ciência que a atividade física estruturada proporciona aos indivíduos que dela se utilizam.

A atividade física é tradicionalmente definida como qualquer movimento corporal produzido pela contração dos músculos esqueléticos que resulte em um aumento substancial em relação ao dispêndio de energia em repouso (ACSM,2010). Com frequência, os termos atividade física e exercício físico são erroneamente utilizados como sinônimos. A American College of Sports Medicine (ACSM) ressalta essa diferença, particularizando o exercício físico como um tipo de atividade física que consiste em movimentos corporais planejados, estruturados e repetitivos realizados para aprimorar ou preservar um ou mais componentes da aptidão física.

A definição apresentada pelo Manifesto do Cirurgião Geral dos Estados Unidos em 1996 e citada por Araújo e Araújo (2000) considera como atividade física todo movimento corporal com gasto energético acima dos níveis de repouso, incluindo atividades decorrentes do dia-a-dia, como tomar banho, vestir-se; atividades realizadas durante o trabalho, como andar, carregar, levantar-se; e também as atividades de lazer, como a prática de exercícios, esportes, danças, etc. Shephard (1990) define atividade física como qualquer movimento corporal produzido pelos músculos esqueléticos que resultem em gasto energético, não se preocupando com a magnitude desse gasto de energia. Esses autores também diferenciam atividade física e exercício físico a partir da intencionalidade do movimento, considerando que o exercício físico é um subgrupo das atividades físicas, que é estruturado, planejado e repetitivo, objetivando a manutenção ou otimização da aptidão física.

A ACSM define aptidão física como um conjunto de atributos ou características que as pessoas possuem ou adquirem e 
ARANA, A.R.A. \& XAVIER, F.B. Qualidade ambiental e promoção de ...

se relacionam com a capacidade de realizar uma atividade física. Esses atributos são subdivididos em "Componentes de Aptidão Física Relacionados à Saúde" e "Componentes da Aptidão Física Relacionados às Habilidades", como vemos no quadro 1.

Quadro 1: Componentes de Aptidão Física Relacionados à Saúde e Relacionados às Habilidades

Componentes de Aptidão Física Relacionados à Saúde e Relacionados às Habilidades

COMPONENTES DE APTIDÃO FÍSICA RELACIONADOS À SAÚDE

- Endurance cardiovascular: Capacidade dos sistemas circulatório e respiratório de fornecerem oxigênio durante uma atividade física sustentada.

- Composição corporal: As quantidades relativas de músculo, gordura, osso e outras partes vitais do organismo.

- Vigor muscular: A capacidade do músculo de exercer força.

- Endurance muscular: A capacidade do músculo de continuar se contraindo sem contrair fadiga.

- Flexibilidade: A amplitude de movimento disponível em uma articulação.

\section{COMPONENTES DA APTIDÃO FÍSICA RELACIONADOS ÀS HABILIDADES}

- Agilidade: A capacidade de modificar a posição do corpo no espaço com velocidade e acurácia.

- Coordenação: A capacidade de utilizar os sentidos, como a vista e a audição, juntamente com outras partes corporais para realizar as tarefas com regularidade e exatidão.

- Balanço (equilíbrio): A manutenção do equilíbrio na condição estacionária ou em movimento.

- Potência: A capacidade ou o ritmo com que se consegue realizar um trabalho.

- Tempo de reação: $\mathrm{O}$ tempo transcorrido entre a estimulação e o início da reação a essa estimulação.

- Velocidade: A capacidade de realizar um movimento dentro de um curto período. 
ARANA, A.R.A. \& XAVIER, F.B. Qualidade ambiental e promoção de ...

Fonte: Adaptado de ACSM's 2010. p2.

Em complemento a essas definições, a questão da aptidão física é abordada por Guedes (1996) em seu capítulo nas "Orientações Básicas sobre Atividades Físicas e Saúde para Profissionais das Áreas de Educação e Saúde", definindo-a como "um estado dinâmico de energia e vitalidade que permite a cada um não apenas a realização das tarefas do cotidiano, as ocupações ativas das horas de lazer e enfrentar emergências imprevistas sem fadiga excessiva, mas, também, evitar o aparecimento das funções hipocinéticas, enquanto funcionando no pico da capacidade intelectual e sentindo uma alegria de viver". Propõe também que a aptidão física seria a capacidade de realizar esforços físicos sem fadiga excessiva, garantindo a sobrevivência de pessoas em boas condições orgânicas no meio ambiente em que vivem.

Dentro desse contexto, a epidemiologia da atividade física é abordada por McArdle (2011) em seu capítulo "Atividade Física, Saúde e Envelhecimento", e definições são aplicadas para caracterizar os padrões de comportamento e suas consequências nos grupos investigados. Para o autor, a terminologia relevante inclui o seguinte:

- Atividade física: Movimento corporal produzido pela contração muscular e que faz aumentar o dispêndio de energia.

- Exercício: Atividade física planejada, estruturada, repetitiva e intencional.

- Aptidão física: Atributos relacionados com a maneira pela qual se executa uma atividade física.

- Saúde: Bem estar físico, mental e social, e não apenas ausência de doença.

A definição de saúde concentra-se no amplo espectro de bem-estar e varia da ausência completa de saúde (quase morte) aos mais altos níveis de função fisiológica. Com frequência, essas definições desafiam nossa maneira de medir e quantificar objetivamente a saúde e a atividade física. Entretanto, elas 
ARANA, A.R.A. \& XAVIER, F.B. Qualidade ambiental e promoção de ...

proporcionam uma ampla perspectiva para estudar o papel da atividade física na saúde e na doença.

Para McArdle (2011), a tendência na avaliação da aptidão física durante os últimos 40 anos deixa de enfatizar os testes que realçam o desempenho motor e a aptidão atlética (i.e., velocidade, potência, equilíbrio e agilidade) e passa a focar as capacidades funcionais, relacionadas com a boa saúde global e com a prevenção de doenças. $\mathrm{O}$ autor define quatro componentes mais comuns da atividade física relacionada com a saúde, sendo estes: aptidão aeróbica e/ou cardiovascular, a composição corporal, a endurance dos músculos abdominais e a flexibilidade da região lombossacra e da musculatura posterior da coxa (hamstring).

Após definirmos atividade física, exercício e aptidão física, esclarecemos mais uma vez que a saúde não se caracteriza apenas como um estado de ausência de doenças, mas como um estado geral de equilíbrio no indivíduo, nos diferentes aspectos e sistemas que caracterizam o homem: biológico, psicológico, social, emocional, mental e intelectual, resultando em sensação de bemestar.

\section{Benefícios da atividade física na saúde}

A investigação sobre a relação entre saúde e atividade física iniciou-se por volta dos anos 20 , com foco em doenças coronárias e estendendo-se posteriormente a estudos dos mais diversos problemas e condições associadas ao sedentarismo. Atualmente existe um número cada vez maior de estudos e documentos que comprovam e relatam os benefícios da aptidão física para a saúde (ARAÚJO \& ARAÚJO, 2000).

A inatividade física possui uma relação causal com quase $30 \%$ de todas as mortes por cardiopatia, câncer de cólon e diabetes (MCARDLE, 2011). As mudanças no estilo de vida poderiam reduzir a mortalidade devida a esses males e aprimorar grandemente as capacidades funcionais cardiovasculares, a qualidade de vida e a vida independente. Sendo assim, os maiores benefícios para a saúde resultariam de estratégias que promovem o 
ARANA, A.R.A. \& XAVIER, F.B. Qualidade ambiental e promoção de ... exercício físico regular. Em qualquer idade, as alterações comportamentais - tornar-se fisicamente mais ativo, deixar de fumar e controlar o peso corporal e a pressão arterial - atuam independentemente, retardando a mortalidade devida a todas as causas. As pessoas com estilo de vida mais saudáveis sobrevivem por mais tempo, e o risco de incapacitação e a necessidade de procurar assistência de saúde no lar é protelada e compactada em um menor número de anos no final da vida (MCARDLE, 2011).

O sedentarismo é apontado como problema de saúde púbica no Brasil. Dados do Instituto Brasileiro de Geografia Estatística (IBGE) em pesquisa divulgada em Dezembro de 2014 apontam que $46 \%$ da população com idade superior a 18 anos é sedentária, não atingindo a recomendação de 150 minutos de atividade física por semana, seja nos momentos de lazer, no trabalho ou nos deslocamentos até o trabalho (PNS, 2014). Ainda segundo a pesquisa, o percentual de pessoas que praticam atividades físicas no lazer diminui de acordo com a faixa etária. De 18 a 24 anos, a proporção é de $35,3 \%$. Essas taxas vão caindo para $25,5 \%$ (de 25 a 39 anos), $18,3 \%$ (de 40 a 59 anos) e 13,6\% (de 60 anos ou mais).

Através da realização de uma revisão de literatura mundial durante os últimos 50 anos, Dr.Frank Booth, cunhador do termo "síndrome de morte ambiental sedentária" (SMSe), concluiu que a inatividade, isoladamente, resulta em uma constelação de problemas e condições capazes de evoluir para a morte prematura. Essa condição de deterioração magistralmente identificada revelou que a SMSe causaria a morte prematura de 2,5 milhões de americanos e custaria 2 a 3 trilhões de dólares em despesas com assistência de saúde nos EUA na década seguinte ao seu estudo (MCARDLE, 2011).

$\mathrm{O}$ autor relaciona a SMSe com as condições de: níveis séricos altos de triacilgliceróis, de colesterol e de glicose, diabetes tipo 2, hipertensão, isquemia miocárdica, arritmias, insuficiência cardíaca congestiva, obesidade, câncer de mama, depressão, dor crônica nas costas, lesão medular, acidente vascular cerebral, caquexia patológica, enfermidades debilitantes, quedas que 
ARANA, A.R.A. \& XAVIER, F.B. Qualidade ambiental e promoção de ...

resultam em quadris fraturados, fraturas vertebrais/femorais. Conclui que se forem feitos esforços destinados à diminuição do tempo em frente ao computador/televisão e acoplados com o aumento da atividade física diária acima da rotina, seria obtido um declínio no número de pessoas que sofrem de síndrome metabólica. Os indivíduos que não participam de nenhuma atividade física moderada ou vigorosa durante as horas de lazer comportam uma probabilidade cerca de duas vezes maior de apresentar a síndrome metabólica que aqueles que se exercitam 150min por semana ou mais (MCARDLE, 2011).

A participação nos exercícios consegue reverter a perda de função, independentemente de quando uma pessoa torna-se fisicamente mais ativa. Um estilo de vida sedentário representa um prognosticador independente e poderoso do risco de doença coronariana e de mortalidade; consequentemente, se os $25 \%$ mais sedentários da população adulta norte-americana forem encorajados a se tornar apenas moderadamente ativos, serão conseguidos benefícios substanciais na esfera de saúde pública (MCARDLE, 2011).

Desde os anos 70 que se sabe que os serviços de saúde são insuficientes, por si sós, para obter ganhos em saúde. A declaração de Alma-Ata há pouco mais de 30 anos, chamava a atenção para o papel das várias instituições e setores de atividade na prevenção da doença e promoção da saúde, principalmente nos países de maior vulnerabilidade econômica e social. De fato, tem-se vindo a verificar ser necessário expandir a avaliação, o planejamento e as ações de saúde pública para além do campo restrito do setor da saúde (SANTANA et al., 2010).

Políticas públicas no país precisam estimular a prática de atividades físicas através de programas que chamem a atenção da população para os seus benefícios na saúde. $\mathrm{O}$ ato de exercitar-se precisa estar incorporado não somente ao cotidiano das pessoas, mas também à cultura popular, aos tratamentos médicos, ao planejamento da família e à educação infantil. Essa necessidade se dá por diferentes fatores: do fator social, quando se proporciona ao 
ARANA, A.R.A. \& XAVIER, F.B. Qualidade ambiental e promoção de ...

homem o direito de estar ativo fisicamente em grupo, ao fator econômico, quando se constata que os custos com saúde individual e coletiva caem em populações fisicamente ativas (ARAÚJO \& ARAÚJO, 2000).

A atividade física pode não representar necessariamente uma "fonte da juventude", porém a maior parte da evidência mostra que quando praticada regularmente ela tem o poder de retardar substancialmente o declínio na capacidade funcional associado ao envelhecimento e ao desuso. Em meio a um quadro crescente de doenças crônicas que atinge a população no país, a cultura da medicalização a que estamos inseridos precisa urgentemente ser substituída pela cultura da promoção e proteção à saúde. Para isso são necessárias iniciativas públicas que não somente informem a população a respeito dos benefícios trazidos pela atividade física, mas também propiciem condições para isso, promovendo ações de incentivo e criando espaços e ambientes que favoreçam a vida ativa.

\section{Qualidade urbana ambiental e saúde}

A qualidade ambiental urbana é um conceito de difícil definição, e está atrelada a vários fatores de infraestrutura, desenvolvimento econômico-social e aqueles ligados à questão ambiental das cidades. Oliveira (1983, apud GOMES; SOARES, 2004) salienta que a qualidade de vida está intimamente ligada à qualidade ambiental, pois vida e meio ambiente são inseparáveis, o que não significa que o meio ambiente determina as várias formas e atividades de vida ou que a vida determina o meio ambiente. $\mathrm{Na}$ verdade, o que existe é uma interação entre ambos que variam de escala em tempo e lugar.

A partir do início da década de 90, parece consolidar-se um consenso entre os estudiosos quanto a dois aspectos relevantes do conceito de qualidade de vida: subjetividade e multidimensionalidade. A subjetividade trata-se de considerar a percepção da pessoa sobre o seu estado de saúde e sobre os 
ARANA, A.R.A. \& XAVIER, F.B. Qualidade ambiental e promoção de ...

aspectos não médicos do seu contexto de vida, ou seja, uma análise que só pode ser feita pelo próprio indivíduo, ao contrário das tendências iniciais de uso do conceito que direcionavam a avaliação a um profissional de saúde. Quanto à multidimensionalidade refere-se ao reconhecimento de que o construto é composto por diferentes dimensões (SEIDL, 2004).

Um estudo feito pela Organização Mundial da Saúde (OMS) em 1994 (UFRGS, 2010), que teve como objetivo a criação de instrumentos medidores de qualidade de vida, classificou-a com um sentido mais amplo. Observou-se uma multidimensionalidade do conceito, surgindo assim uma estrutura composta por seis domínios:

- domínio I (físico): dor, desconforto, energia, fadiga, sono e repouso;

- domínio II (psicológico): sentimentos positivos, autoestima, aparência, sentimentos negativos, etc.;

- domínio III (nível de independência): mobilidade, atividades da vida cotidiana, uso de medicação e tratamentos, capacidade de trabalho/produção, etc.;

- domínio IV (relações sociais): relações interpessoais, apoio social, atividade sexual, etc.;

- domínio V (ambiente): segurança física e proteção, recursos financeiros, lazer, ambiente físico - poluição, ruído, trânsito, clima - etc.;

- domínio VI (aspectos espirituais, crenças pessoais ou religião): espiritualidade, religião e crenças pessoais.

Nesse caso, percebe-se claramente que o ambiente - domínio $\mathrm{V}$, com destaque para lazer, além do ambiente físico propriamente dito - torna-se um elemento relevante para a avaliação da qualidade de vida, principalmente no contexto urbano, no qual a poluição do ar, a sonora e a visual podem influenciar diretamente no bem-estar da população (UFRGS, 2010).

No entanto, o tema qualidade ambiental é bastante complexo, bem como seus padrões e seus indicadores. Fatores subjetivos que levam em conta a percepção que o indivíduo tem 
ARANA, A.R.A. \& XAVIER, F.B. Qualidade ambiental e promoção de ...

em relação ao seu ambiente e ao seu próprio modo de vida estão contidos nesses indicadores (SILVA, 2002). Além disso, existem os fatores objetivos: econômicos, sociais, culturais e políticos, que se manifestam distintamente no espaço (SILVA, 2002), possibilitando diversos tipos de interpretações, e tornando difícil de chegar a uma conclusão.

A despeito dessa dificuldade, há, porém um consenso quando se relaciona qualidade ambiental urbana e vegetação. Considera-se que a qualidade ambiental satisfatória é determinada por composições paisagísticas que privilegie, sobretudo, mas não somente, a vegetação; vista desde um simples gramado às mais diversas espécies arbóreas. Nessas condições, a vegetação constitui componente chave, embora outros componentes também sejam necessários para o alcance de um padrão mínimo de qualidade do ambiente (GOMES; SOARES, 2004).

Sobre essa relação, Richter \& Bocker afirmam:

A qualidade ambiental urbana está diretamente ligada ao acesso dos moradores à quantidade, qualidade $\mathrm{e}$ distribuição de espaços livres de construção que possam permitir um saudável contato com a natureza, propiciando também possibilidades de socialização e expressão cultural; portanto, uma combinação entre conservação da natureza, conservação da flora e da fauna, conservação do solo, funções climáticas e as necessidades da população em relação à recreação e relaxamento em contato com a natureza. Nesse caso, é essencial a implantação de espaços livres urbanos que possam satisfazer os diversos interesses humanos das mais variadas formas (RICHTER; BÖCKER, 1998, p57).

A manutenção das áreas verdes urbanas sempre foi justificada pelo seu potencial em propiciar qualidade ambiental à população. Ela interfere diretamente na qualidade de vida dos seres por meio das funções sociais, ecológicas, estéticas e educativas que elas exercem para amenização das consequências negativas da 
ARANA, A.R.A. \& XAVIER, F.B. Qualidade ambiental e promoção de ... urbanização (CAPORUSSO; MATIAS, 2008). O impacto do espaço verde sobre a saúde é cada vez mais reconhecido.

Para Milano (1990, apud CAPORUSSO; MATIAS, 2008) a principal função do sistema de áreas verdes urbanas não deve ser apenas a criação de refúgios para que as pessoas possam "escapar" da cidade. Além disso, estas áreas devem possibilitar à população momentos de lazer e recreação em contato com a natureza, respeitando sua vivência urbana e contato com outras pessoas.

A oferta de espaços verdes seguros, limpos e confortáveis tem impactos na saúde, medidos de forma direta através do estado de saúde auto avaliado e, de forma indireta, através da melhoria da qualidade ambiental. Dado o crescente consenso que o ambiente é a chave em promover expansão da energia, garantir oportunidades para o aumento da atividade física é um meio promissor de combate ao comportamento sedentário associado com uma variedade de doenças crônicas.

Alguns planejamentos urbanos desconsideram o papel multifuncional desses espaços verdes e muitas decisões são baseadas apenas em estética e custo. O que falta em muitos casos é uma visão abrangente do papel dos parques urbanos e a compreensão de que eles formam uma estrutura que pode resultar em qualidade de vida e constituir uma chave na estratégia de saúde proposta por autoridades.

\section{Fatores para o uso ativo de parques urbanos}

Os fatores utilizados para medir o uso dos parques variam em termos de período, tempo de atividade, tipos de parques estudados, entre outros. Isso produz estimativas igualmente variadas de prevalência de seu uso. Mensurar o nível de atividade física realizada nos parques e relevância dos mesmos na estratégia de promoção de saúde através de uma população mais ativa tornase um desafio multidisciplinar.

Nos Estados Unidos e na Austrália as atividades passivas realizadas nos parques superam a prática de atividade física regular 
ARANA, A.R.A. \& XAVIER, F.B. Qualidade ambiental e promoção de ...

(GILES-CORTI et al., 2005). Na Austrália, por exemplo, a prevalência do uso adulto de parques para a atividade física nas duas semanas anteriores ao estudo variou de $13,0 \%$ a $17,3 \%$ do total de frequência do parque (GILES-CORTI et al., 2005). Já no Brasil, um estudo realizado por Reis (2001) na cidade de Curitiba constatou que a maioria da população presente no Jardim Botânico encontra-se fisicamente ativa, e ainda que $61,85 \%$ desses indivíduos atingem as recomendações para a prática de atividade física. À proporção disso, Brownson (2000, apud REIS, 2001) constatou que em 50 estados americanos somente $38 \%$ da população analisada atingiu as mesmas recomendações.

Inúmeros fatores observáveis podem influenciar o uso dos parques urbanos. Uma revisão da literatura por Broomhall (2005, apud GILES-CORTI et al., 2005) incluiu a esses: as características do próprio parque - qualidade e quantidade do espaço disponível; o acesso a instalações locais - quadras e centros de lazer; a relação entre atributos do parque e necessidades dos usuários locais; a manutenção do parque e ainda os fatores de segurança percebida.

Em sua proposta conceitual, Bendimo-Rung et al. (2005) propõem um modelo para guiar pensamentos e sugerir hipóteses, e define alguns atributos dos parques que podem ser utilizados como padrão para futuras pesquisas. Nesta classificação, a discussão centra-se sobre as características ambientais do parque que poderiam estar relacionados à atividade física, sendo essas as características do ambiente, condições dos parques, facilidade ou dificuldade de acesso, estética do local, segurança percebida e políticas voltadas a esse objetivo. De outra maneira, Tinsley et al. (2002) atribui o uso dos parques a fatores como proximidade, acessibilidade (não existência de rodovias), aspectos estéticos como a presença de árvores, água (lago) e existência de pássaros, manutenção do parque, tamanho e a disponibilidade de facilidades como calçadas ou percursos para pedestres (caminhada).

Em meio à diversidade de variáveis, existe certo consenso encontrado em um número significativo de pesquisas ao se tratar da relação positiva entre o fácil acesso e/ou proximidade a parques 
ARANA, A.R.A. \& XAVIER, F.B. Qualidade ambiental e promoção de ...

urbanos e o aumento do nível de atividade física nesses locais (REIS, 2001; GILES-CORTI et al., 2005; COHEN et al., 2007; KACZYNSKI AND HENDERSON, 2007; KACZYNSKI et al., 2008; SUGIYAMA et al., 2010; COOMBES, 2010; VEITCH et al., 2013). Em estudo recente realizado nos Estados Unidos, participantes relataram-se propensos a visitar parques locais desde que não seja necessário atravessar ou viajar em estrada de tráfego intenso (KACZYNSKI, 2014, apud VEITCH 2014).

A despeito disso, ao explorar a disponibilidade de acesso a parques urbanos e sua relação com a prática de atividades físicas, muitos autores não encontraram relação alguma em Norwich, na Inglaterra (HILLSDON, 2006), nos parques da Georgia e Missouri nos Estados Unidos (HOEHNER et al., 2005), em estudo nacional realizado na Nova Zelândia (WITTEN et al., 2008) e também na Escócia (ORD et al., 2013). Esses autores partem da premissa que os parques urbanos são influentes na saúde das populações estudadas, mas desconsideram que a prática de atividade física seja o mecanismo que explique essa associação. Esses resultados adversos dificultam a elucidação da temática em questão.

A estética e características do parque também têm sido positivamente associadas à visitação do parque para a manutenção da vida ativa (GILES-CORTI et al., 2005; REIS et al., 2009). Ao revisar 18 estudos, Owen et al. (2004) concluiu que fatores estéticos e a presença colinas estavam diretamente associados a atividades físicas auto percebidas, apesar de nenhum desses estudos reportar qual o tipo de atividade física que ocorreu nesses locais (COHEN et al., 2007). Na cidade de Curitiba, a beleza geográfica do parque e as pistas de caminhada/corrida foram atribuídas como incentivadoras da prática de atividade física por mais de 94\% dos entrevistados (REIS, 2001).

O tamanho dos parques e a proximidade a parques "formais" foram considerados por Coombes et al. (2010) características essenciais na busca de níveis de atividade física e diminuição do sobrepeso. No que concerne a isso, Cohen et al. (2007) concluíram que recursos, mais do que o tamanho do parque, atraem pessoas para 
ARANA, A.R.A. \& XAVIER, F.B. Qualidade ambiental e promoção de ... a prática de atividades físicas, incluindo acessibilidade, disponibilidade e qualidade das facilidades. Kaczynski et al. (2008), por sua vez, classifica a presença de trilhas - pavimentadas, não pavimentadas e arborizadas - como os determinantes da prática em questão, e desconsidera a influência da estética e segurança do parque nos 33 parques analisados no Canadá.

No Brasil, um estudo feito em Florianópolis por Colett et al. (2008) observou-se que os equipamentos disponíveis no parque não se apresentaram como fatores relevantes, contrariando os achados de Cohen et al. (2007). A mesma escala de auto percepção do ambiente utilizada por Colett et al. (2008) foi posteriormente utilizada por Silva; Petroski e Reis (2009) em Curitiba. Curiosamente, o indicador "equipamentos disponíveis" se apresentou como fator fortemente estimulador para a prática de atividades físicas. Observou-se que no segundo estudo, a população que reportou esse fator como estimulante advinha de famílias com baixo nível sócio econômico. Isso pode indicar que quanto mais baixa a renda do indivíduo, maior a tendência em perceber esse fator como estimulador. Uma possível explicação seria que pessoas de baixa renda têm poucas oportunidades de praticar atividades físicas em locais particulares (clubes e academias) e valorizam ambientes públicos com equipamentos disponíveis gratuitamente (SCOTT; JACKSON, 1996; BEDIMORUNG et al., 2005; GORDON-LARSEN et al., 2006; POWELL et al., 2006 apud SILVA; PETROSKI; REIS, 2009).

Quanto ao fator tecnológico e arquitetônico do ambiente no parque, os resultados encontrados em Florianópolis (COLLET et al.,2008) também diferiram quando comparados aos de Curitiba (SILVA; PETROSKI; REIS, 2009). Enquanto Silva; Petroski e Reis (2009) observaram que em Curitiba a pista de caminhada no parque, estacionamento e beleza arquitetônica das estruturas construídas foram fatores estimulantes para a população analisada, Colett et al. (2008) concluiu que no parque de Florianópolis podem ser considerados estímulos positivos somente a estética 
ARANA, A.R.A. \& XAVIER, F.B. Qualidade ambiental e promoção de ...

arquitetônica das estruturas construídas e a pista de caminhada e corrida, sendo considerado indiferente o estacionamento no parque.

Outro estudo realizado em Curitiba associou qualidade ambiental à frequência no uso de parques e praças (CASSOU, 2009). A maioria dos usuários dos parques estudados indicou ser a beleza e presença de estacionamento os fatores mais significativos. Já os frequentadores das praças consideram imprescindíveis a proximidade de casa, estrutura, equipamentos e segurança percebida.

Embora muitos estudos incluam a vegetação presente nos parques no grupo dos "fatores estéticos", outros estudos buscam esclarecer essa relação de maneira mais específica. Em 1999, estudiosos da Filadélfia iniciaram um programa para transformar lotes vagos da cidade em ambientes verdes. Estudos analisaram a saúde e a segurança da população durante os 10 anos da intervenção, e também compararam o resultado final com outros locais vagos onde o programa não havia sido implantado (BRANAS et al., 2011). Os resultados da pesquisa sugeriram que a "verdificação" do lote foi suficiente para reduzir a criminalidade e promover alguns aspectos de saúde. Ademais, alguns pontos que sofreram essa transformação obtiveram significante aumento na prática de exercício físico e também na diminuição no stress (BRANAS et al., 2011). Contudo, o fato de esse aumento só haver alcançado pontos isolados da cidade sugere que normas culturais e/ou sociais podem relacionar-se mais intimamente à prática de exercícios físicos quando comparadas à presença de vegetação urbana.

Ainda em relação à vegetação, Maas et.al (2008) através de estudo realizado na Nova Zelândia concluem que a quantidade de espaço verde no ambiente de vida é dificilmente relacionada com o nível de atividade física. Para os autores, a quantidade de atividade física realizada em ambientes de vida mais ecológicos não explica a relação entre o espaço verde e saúde.

Isso posto, a presença de espaços verdes públicos nas cidades mostra-se fundamental para a qualidade ambiental e saúde da população, mas somente o fato de existirem não parece garantir os objetivos propostos por essa pesquisa. À essa presença atrelam- 
ARANA, A.R.A. \& XAVIER, F.B. Qualidade ambiental e promoção de ...

se tantos outros fatores supracitados, a fim de que esses ambientes atinjam o nível de atividade física necessário para obtenção de ganhos em saúde.

\section{Determinantes individuais para o uso ativo de parques urbanos}

A utilização dos parques não depende apenas de atributos que o local oferece, mas também se relacionam com preferências individuais como idade, hábitos de exercício e raça/etnia (COHEN et al., 2007). Broomhall (2005) destaca as características dos potenciais utilizadores como de extrema importância para sua adesão à atividade física nesses ambientes, enumerando-as por: status socioeconômico, idade, gênero, etnia, além de fatores psicológicos como auto eficácia e barreiras individuais que influenciam as preferências pessoais.

Em proposta conceitual, Bedimo-Rung et al. (2005) enumeram também as razões pessoais mais comuns para não se envolver em atividades nos parques, que incluem a falta de tempo, dinheiro, saúde pessoal, informação, transporte e acesso, preocupações de segurança e também a falta de companheiros de lazer.

Os resultados encontrados até o presente momento, apesar de variados, apontam alguns perfis e preferências em questão. Bedimo-Rung et al. (2005) constataram que os adultos mais velhos, mulheres e as famílias de renda mais baixa são menos propensos a frequentarem os parques públicos para a prática de atividade física. A despeito disso, Reis (2001) aponta resultados diferentes no Jardim Botânico em Curitiba: igualdade na distribuição entre homens e mulheres e predomínio de adultos acima de 35 anos. Posteriormente, também em Curitiba, Cassou (2009) constatou que quanto maior a qualidade ambiental dos parques e praças, maior é a utilização destes por mulheres, idosos e pessoas escolarizadas.

Hillsdon et al. (2006), assim como Bedimo-Rung et al. (2005) também encontrou na Inglaterra uma diferença significante de gêneros 
ARANA, A.R.A. \& XAVIER, F.B. Qualidade ambiental e promoção de ...

fisicamente ativos nos parques de Norwich: os homens são duas vezes mais ativos que as mulheres. Cohen et al. (2007), que também observou essa diferença, chamou a atenção para a ausência de mulheres em atividades vigorosas. Os autores observaram a presença de mulheres em atividades sedentárias ou em locais que estivessem observando crianças, sendo pouco presentes em campos, quadras e locais de jogos competitivos. Chama a atenção o fato de essa diferença significativa de gênero ativo não ter sido encontrada nos parques analisados no Brasil (Florianópolis e Curitiba), o que sugere que as mulheres brasileiras podem estar mais conscientes quanto a necessidade de exercícios físicos no dia a dia.

$\mathrm{O}$ fator valores e atitudes foram considerados estimulantes em parques de Florianópolis, uma vez que os indicadores de comportamento frequentemente observados dos usuários, apoio/incentivo de amigos e imagem atribuída pela comunidade, foram declarados positivamente associados à realização de atividades físicas no local (COLLET et al, 2008). Os resultados encontrados confirmam as evidências de outros estudos que indicam que há associação positiva entre atividades físicas em parques e aspectos como o apoio e estímulo vindo de familiares e amigos, além do contato com demais pessoas que também realizam atividades físicas (COLLET et al, 2008), sugerindo que observar pessoas praticando atividades físicas pode constituir um fator intrinsicamente estimulante para a auto percepção de saúde do indivíduo e busca por uma vida mais ativa.

$\mathrm{O}$ fator econômico da população no local em que o parque se insere também demonstra influência na questão estudada. O nível social do bairro determina muitas vezes a acessibilidade aos parques, combinando fator individual com determinante físico. Veitch et al. (2014) constataram que adolescentes que vivem em bairros de classe social mais baixa costumam ter que se deslocar duas vezes mais do que os adolescentes de classe alta para chegar ao parque mais próximo, sugerindo que essa disparidade dificulte a frequência dos primeiros. 
ARANA, A.R.A. \& XAVIER, F.B. Qualidade ambiental e promoção de ...

Sendo assim, qualidade ambiental urbana e o uso dos parques para a prática de atividades físicas atrelam-se à subjetividade. Muitos são os determinantes que incutem motivações intrínsecas ao ser humano na busca de saúde através desses espaços. A diversidade de população e localizações geográficas apresentados nesse estudo sugere que a própria distribuição desigual de espaços públicos abertos em todas as cidades e entre os países podem explicar as variações interculturais e sócias econômicas abordadas.

\section{Considerações finais}

Desafios logísticos e até mesmo custos financeiros dificultam a pesquisa que envolve a relação entre parques urbanos e prática de atividade física, que ainda é reduzida. Muitas são as barreiras encontradas na tentativa de traçar um perfil dos frequentadores bem como enumerar as características desses ambientes que atraem pessoas para seu uso ativo. Essa dificuldade pode estar relacionada à diversidade de metodologias utilizadas, à indefinição de qualidade dos espaços, às medidas e definições de espaço verde, às diferentes populações e localizações geográficas abordadas, bem como aos instrumentos de auto relato de atividade física que nem sempre expõem a realidade.

Esta revisão demonstrou que a utilização de parques urbanos possibilita melhoras na qualidade de vida da população. O fator "proximidade" e a "estética" dos parques parecem ser os principais determinantes para a adesão e manutenção de frequentadores assíduos. Nesse sentido, compreende-se que a disponibilidade de parques não garante a promoção da atividade física se não forem consideradas também o perfil e as necessidades da comunidade que os cercam. É necessário que os parques apresentem facilidade de acesso, qualidade e quantidade de infraestrutura, belezas naturais entre outros fatores, para que as pessoas se sintam atraídas e motivadas a frequentá-los. 
ARANA, A.R.A. \& XAVIER, F.B. Qualidade ambiental e promoção de ...

No Brasil, ainda são raras as publicações que dissertam sobre a importância da qualidade dos parques urbanos para a atividade física (REIS, 2001; COLLET et al., 2008; SILVA; PETROSKI; REIS, 2009; CASSOU, 2009), sendo essas concentradas na região sul do país. Essa escassez torna difícil a compreensão do potencial benefício que os espaços verdes urbanos podem trazer à saúde da população brasileira.

Os artigos que abordam essa questão no país trouxeram resultados dissidentes da pesquisa realizada pelo IBGE em 2014, já que a população mais ativa encontrada nos parques estudados foi a população adulta até 34 anos em Florianópolis e acima de 35 anos em Curitiba, e por sua vez a pesquisa do Instituto Brasileiro constatou que o nível de atividade física diminui com a idade. Esses resultados podem sugerir que a população mais jovem brasileira seja fisicamente ativa em outros locais que não sejam os parques urbanos, como também podem incutir uma realidade própria dos parques estudados, que por diversos fatores econômicos e sociais pode refletir uma verdade diferente do restante do país.

Além disso, três dos quatro estudos realizados no Brasil que foram encontrados pelos pesquisadores desse artigo fizeram uso do mesmo instrumento metodológico, a escala de auto percepção do ambienta validada por Reis (2002). Outros instrumentos devem ser utilizados e a pesquisa nesse ramo precisa ser estimulada, para que uma estratégia multinível que combine indivíduo e meio ambiente possa finalmente atingir as metas que o seu potencial econômico, social e ambiental tem a capacidade de abranger.

\section{Referências bibliográficas}

ACSM's - Guidelines for Exercise Testing and Prescription. Philadelphia: Lippincott Williams \& Wilkins, 8th edition, 2010. Capítulo I, p2. 
ARANA, A.R.A. \& XAVIER, F.B. Qualidade ambiental e promoção de ...

ARAÚJO, D.S.M.S.; ARAÚJO, C.G. S. Aptidão física, saúde e qualidade de vida relacionada à saúde em adultos. Revista Brasileira de Medicina do Esporte, Niterói, v. 6, n. 5, p.194-203, out. 2000.

BEDIMO-RUNG AL, MOWEN AJ, COHEN DA. The significance of parks to physical activity and public health: a conceptual model. Am J Prev Med. 2005; 28:159-168.

BRANAS CC, CHENEY RA, MACDONALD JM, TAM VW, JACKSON TD, HAVE TRT. A Difference-in-Differences Analysis of Health, Safety, and Greening Vacant Urban Space. American Journal of Epidemiology. 2011.

CAPORUSSO, D. MATIAS, L.F. Áreas Verdes Urbanas: Avaliação e Proposta Conceitual. Simpósio de Pós graduação em Geografia de Estado de São Paulo - SIMPGEO-SP. 17 à 19 de Dezembro de 2008, Rio Claro/SP. 2011.

CASSOU, A.C.N. Características ambientais, Frequência de utilização e nível de atividade física dos usuários de parques e praças de Curitiba-PR. Curitiba, 2009. 130 p. Dissertação (Mestrado em Educação Física) - Universidade Federal do Paraná.

COHEN DA, MCKENZIE TL, SEHGAL A, WILLIAMSON S, GOLINELLI D, LURIE N. Contribution of public parks to physical activity.AmJ Public Health 2007;97:509 -14.

COLLET C, CHIARADIA BM, REIS RS, NASCIMENTO JV. Fatores determinantes para a realização de atividades físicas em parque urbano de Florianópolis. Rev Bras Ativ Fís Saúde 2008. 13(1):15-23.

COOMBES E., JONES A.P., HILLSDON M. The relationship of physical activity and overweight to objectively measured green 
ARANA, A.R.A. \& XAVIER, F.B. Qualidade ambiental e promoção de ...

space accessibility and use. Social Science and Medicine. 2010;70:816-822.

GILES-CORTI, B., BROOMHALL, M., KNUIMAN, M., COLLINS, C., DOUGLAS, K., et al., 2005. Increasing walking: how important is distance to, attractiveness, and size of public open space? American Journal of Preventive Medicine 28, 169-176.

GOMES MAS, SOARES BR. Reflexões sobre qualidade ambiental urbana. Estudos Geográficos, Rio Claro, 2(2): 21-30 ,jul-dez - 2004 (ISSN 1678-698X).

GUEDES, D.P. Atividade física, aptidão física e saúde. In: CARVALHO, T.; GUEDES, D.P.; SILVA, J.G. (Orgs.). Orientações Básicas sobre Atividade Física e Saúde para Profissionais das Áreas de Educação e Saúde. Brasília: Ministério da Saúde e Ministério da Educação e do Desporto, 1996.

HILlSDON, M, PANTER, J, FOSTER, C et al., (2006). The relationship between access and quality of urban green space with population physical activity. Public health, 120 (12), 1127-1132.

HOEHNER, C.M.; BRENNAN RAMIREZ, L.K.; ELLIOTT, M.B.; HANDY, S.L.; BROWNSON, R.C. Perceived and objective environmental measures and physical activity among urban adults. American Journal of Preventive Medicine. Amsterdam, v. 28, n. 2, suplemento 2, p. 105-116, 2005.

MAAS J, VERHEIJ RA, SPREEUWENBERG P, GROENEWEGEN PP. Physical activity as a possible mechanism behind the relationship between green space and health: a multilevel analysis. BMC Public Health 2008.

MCARDLE, WILLIAM D. Fisiologia do exercício: nutrição, energia e desempenho humano / William D. McArdle, Frank I. 
ARANA, A.R.A. \& XAVIER, F.B. Qualidade ambiental e promoção de ...

Katch, Victor L. Katch; traduzido por Giuseppe Taranto. Rio de Janeiro: Guanabara Koogan, 2011. Il.

KACZYNSKI A, POTWARKA L, SAELENS B. Association of park size, distance, and features with physical activity in neighbourhood parks. Am J Public Health 2008;98:1451-6.

KACZYNSKI, A.T., HENDERSON, K.A., 2007. Environmental correlates of physical activity: a review of evidence about parks and recreation. Leis. Sci. 29, 315-354.

KACZYNSKI AT, KOOHSARI MJ, STANIS SA, BERGSTROM R, SUGIYAMA T: Association of street connectivity and road traffic speed with park usage and park-based physical activity. Am J Health Prom 2014, 28:197-203.

REIS R, HINO A, FLORINDO A, ANEZ C, DOMINGUES M. Association between physical activity in parks and perceived environment: a study with adolescents. J Phys Act Health 2009;6:503-9.

ORD K, MITCHELL R, AND PEARCE J. Is level of neighbourhood green space associated with physical activity in green space? International Journal of Behavioural Nutrition and Physical Activity. 2013. 10: 127.

OWEN N, HUMPEL N, LESLIE E, BAUMAN A, SALLIS JF. Understanding environmental influences on walking: review and research agenda. Am J Prev Med. 2004;27:67-76.

PESQUISA NACIONAL DE SAÚDE: 2013 : Percepção do estado de saúde, estilos de vida e doenças crônicas : Brasil, grandes regiões e unidades da federação. Rio de Janeiro. IBGE. 2014. 
ARANA, A.R.A. \& XAVIER, F.B. Qualidade ambiental e promoção de ...

PNS- Pesquisa Nacional de Saúde. Percepção do estado de saúde, estilo de vida e doenças crônicas.- Brasil, Grandes Regiões e Unidades da Federação [Internet]. Rio de Janeiro: Instituto Brasileiro de Geografia e Estatística; 2014 [citado 2015 fev 9]. 181 p. Disponível em: ftp://ftp.ibge.gov.br/PNS/2013/pns2013.pdf

REIS RS. Determinantes ambientais para a realização de atividades físicas nos parques urbanos de Curitiba: uma abordagem sócio ecológica da percepção dos usuários. [Dissertação de Mestrado]. Florianópolis: Centro de Desportos - Universidade Federal de Santa Catarina; 2001.

RICHTER, M.; BÖCKER, R. Developing an urban landscape management concept through the integration of environmental quality goals and environmental information systems. In: BREUSTE, J.; FELDMANN, H.; UHLMANN, O. (Orgs.) Urban ecology. Berlim: Springer, 1998. 223p.

SANTANA, P. et. al. O papel dos espaços verdes urbanos no bemestar e saúde das populações. Lisboa: [s.n.], 2010.

SEIDL, ELIANE MARIA FLEURY; ZANNON, CÉLIA MARIA LANA DA COSTA. Qualidade de vida e saúde: aspectos conceituais e metodológicos. Cad. Saúde Pública, Rio de Janeiro , v. 20, n. 2, Apr. 2004.

SHEPHARD RJ, Bouchard C. Principal components of fitness: relationship to physical activity and lifestyle. Can J Appl Physiol 1994;19:200-14.

SILVA, M.L.G. da. Análise da Qualidade Ambiental Urbana da Bacia Hidrográfica da Lagoa da Conceição. Florianópolis, 2002. 111f. Dissertação (Mestrado em Engenharia de Produção) Programa de Pós-Graduação em Engenharia de Produção, UFSC. 
ARANA, A.R.A. \& XAVIER, F.B. Qualidade ambiental e promoção de ...

SILVA, D. A. S.; PETROSKI; E. L.; REIS, R. S. Barreiras e facilitadores de atividades físicas em freqüentadores de parques públicos. Motriz, Rio Claro, v.15, n. 2, p. 219-227, abr./jun. 2009.

SUGIYAMA T., FRANCIS J., MIDDLETON N.J., OWEN N., GILES-CORTI B. Associations between recreational walking and attractiveness, size, and proximity of neighborhood open spaces. American Journal of Public Health. 2010;100(9):1752-1757.

TINSLEY H., TINSLEY, D., AND CROSKEYS, C. Park usage, social milieu and psychosocial benefits of park use reported by older urban park users from four ethnic groups. Leisure Sci. 2002; 24: 199-218.

UNIVERSIDADE FEDERAL DO RIO GRANDE DO SUL. Psiquiatria. Divisão de

Saúde Mental. Grupo WHOQOL. 2006.

VEITCH J, BALL K, CRAWFORD D, ABBOTT G, SALMON J. Is park visitation associated with leisure-time and transportation physical activity? Prev Med. 2013 Nov; 57(5):732-4.

VEITCH, JENNY, CARVER, ALISON, HUME, CLARE, CRAWFORD, DAVID, TIMPERIO, ANNA, BALL, KYLIE AND SALMON, JO. 2014, Are independent mobility and territorial range associated with park visitation among youth?, International journal of behavioral nutrition and physical activity, vol. 11, no. 1, Article number 73, pp. 1-12.

WITTEN $\mathrm{K}$, HISCOCK $\mathrm{R}$, PEARCE J, BLAKELY $\mathrm{T}$. Neighbourhood access to public open space and the physical activity of residents: a national study. Preventive Medicine, 2008. 47 (3) $299-303$. 
ARANA, A.R.A. \& XAVIER, F.B. Qualidade ambiental e promoção de ...

Recebido em novembro de 2013 Aceito em dezembro de 2016 